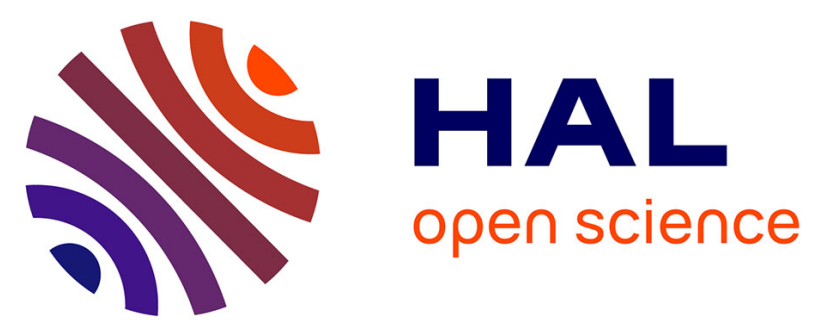

\title{
A FastSLAM Approach Integrating Beamforming Maps for Ultrasound-based Robotic Inspection of Metal Structures
}

Othmane-Latif Ouabi, Pascal Pomarede, Matthieu Geist, Nico F Declercq, Cedric Pradalier

\section{To cite this version:}

Othmane-Latif Ouabi, Pascal Pomarede, Matthieu Geist, Nico F Declercq, Cedric Pradalier. A FastSLAM Approach Integrating Beamforming Maps for Ultrasound-based Robotic Inspection of Metal Structures. 2021. hal-03017841v2

\section{HAL Id: hal-03017841 \\ https://hal.science/hal-03017841v2}

Preprint submitted on 19 Jan 2021 (v2), last revised 19 Nov 2021 (v3)

HAL is a multi-disciplinary open access archive for the deposit and dissemination of scientific research documents, whether they are published or not. The documents may come from teaching and research institutions in France or abroad, or from public or private research centers.
L'archive ouverte pluridisciplinaire HAL, est destinée au dépôt et à la diffusion de documents scientifiques de niveau recherche, publiés ou non, émanant des établissements d'enseignement et de recherche français ou étrangers, des laboratoires publics ou privés. 


\title{
A FastSLAM Approach Integrating Beamforming Maps for Ultrasound-based Robotic Inspection of Metal Structures
}

\author{
Othmane-Latif Ouabi ${ }^{1}$, Pascal Pomarede ${ }^{1}$, Matthieu Geist ${ }^{2}$, Nico F. Declercq ${ }^{1,3}$, Cédric Pradalier ${ }^{1}$
}

\begin{abstract}
We present a novel FastSLAM approach for a robotic system inspecting structures made of large metal plates. By taking advantage of the reflections of ultrasonic guided waves on the plate boundaries, it is possible to recover, with enough precision, both the plate shape and the robot trajectory. Contrary to our previous work, this approach takes into account the dispersive nature of guided waves in metal plates. This is leveraged to construct beamforming maps from which we solve the mapping problem through plate edges estimation for every particle, in a FastSLAM fashion. It will be demonstrated, with real acoustic measurements obtained on different metal plates, that such a framework achieves better results in terms of convergence and accuracy, while the complexity of the algorithm is sensibly reduced.
\end{abstract}

\section{INTRODUCTION}

In this work $^{1}$, we describe a new FastSLAM approach [1] to achieve Simultaneous Localization and Mapping (SLAM) for a robotic system relying on Ultrasonic Guided Waves (UGWs) to support inspection tasks on large metal structures such as storage tanks or ship hulls. In Structural Health Monitoring (SHM), acoustic tomography techniques can be used for defect detection and characterization, but they rely on the accurate prior knowledge of the positions of the sensors which are integrated into the structure [2], [3]. To deploy similar methods on a robotic platform, recovering the robot position with respect to the individual metal plates may be beneficial, as it could lead, in combination with external localization systems, to precise localization of the mobile unit, and thus, to accurate inspection results.
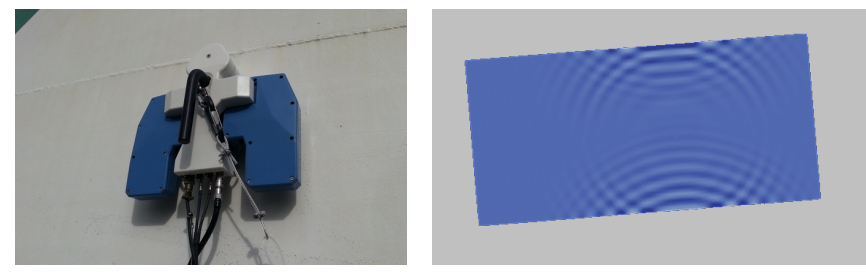

Fig. 1. (Left) A magnetic crawler carrying out an inspection task on a metal structure. (Right) Guided waves reflected by the edges of a plate in a simulation environment. We aim to enable on-plate localization and mapping with a high precision for magnetic crawlers equipped with acoustic transducers, and relying on such ultrasonic reflections.

\footnotetext{
${ }^{1}$ Othmane-Latif Ouabi, Pascal Pomarede, Nico F. Declercq and Cédric Pradalier are with the GeorgiaTech Lorraine and the UMI2958 GT-CNRS in Metz, France. firstname.lastnamedgeorgiatech-metz.fr

${ }^{2}$ Matthieu Geist is with Google Research, Brain Team. mfgeistegoogle.com

${ }^{3}$ Nico F. Declercq is also with Georgia Institute of Technology, Atlanta, GA 30332-0250, USA. declercqegatech.edu

${ }^{1}$ This work is part of the BugWright2 project. This project is supported by the European Commission under grant agreement 871260 - BugWright2.
}

On metal plates, guided waves are often generated by applying piezo-electric transducers in contact with the surface. These waves propagate radially around the emitter through the plate material, and potentially over large distances. When encountering the plate edges, these waves are reflected perpendicularly, and a receiver can sense the reflections. In this setup, the resulting acoustic data carry essential information on the source position and the plate geometry.

In this work, we consider a mobile unit equipped with acoustic transducers for both emission and reception, and moving on a metal surface. We leverage the sensing of the ultrasonic reflections to estimate both the plate shape and the robot trajectory. The principle of this approach is illustrated in Fig.1. In the robotic field, this problem is known as Simultaneous Localization and Mapping (SLAM).

One of the significant challenges arises from the dispersive nature of UGWs [4]. It means that the propagation velocity is a function of the wave frequency, resulting in a waveform deformation when the propagation distance increases. Besides, propagation in metal plates is highly reverberant. These characteristics account for the relative complexity of acoustic data and call for specific processing methods to achieve onplate localization and mapping with high accuracy. On the robotic aspect, recent works consider the similar problem of room shape reconstruction from acoustic echoes [5], [6]. As the sound velocity in the air is constant, the determination, from the measurements, of the first-order reflections is not a significant issue. However, identifying several echoes from guided wave data is more difficult due to the wave dispersion and the wave packets overlapping.

In our previous work [7], ultrasonic measurements on metal plates have proven to yield sufficient information to provide both localization and mapping capabilities on metal plates. However, the dispersive nature of the waves was not taken into account and the relative complexity of the algorithm may jeopardize its robustness and accuracy. In this paper, we present an alternative method to solve the SLAM problem from ultrasonic measurements. First, a wave propagation model is introduced and is leveraged to detect acoustic reflections. From them, we build beamforming maps [8] which are subsequently integrated into a FastSLAM framework to solve the mapping problem. Our approach achieves more accurate results than our previous method on real measurements, with less algorithmic complexity.

In summary, our contributions are the introduction of propagation models and the integration of beamforming maps in FastSLAM to achieve on-plate Simultaneous Localization and Mapping with high accuracy for robotic inspection. 


\section{RELATED WORK}

On the one hand, standard methods to inspect large metal structures consist in deploying a mobile robot to perform point-by-point thickness measurements with an acoustic probe, but the entire surface cannot be inspected in a reasonable amount of time due to the limited surface of the transducer. On the other hand, UGWs have been successfully used by SHM systems to inspect large structures such as pipelines or rails [4], [9], but the transducers are integrated into the structure and their position is known accurately. Hence, outside of the authors' works, UGWs-based techniques have not been deployed on a robotic system, nor have guided waves proven to yield accurate localization capabilities which are critical for such methods to work.

Moreover, UGWs propagation is dispersive, which means that the longer the distance a wave packet travels in a metal plate, the more it deforms. Fig. 2 illustrates this phenomenon. It shows that the shape of the signal is significantly different after propagating over two meters. In SHM, the chosen frequency range generally lies in a dispersion-limited bandwidth, but for our case-study, waves might propagate over much larger distances. Hence, wave dispersion may still have some effects on the signals, and shall not be neglected. In the literature, the use of propagation models in the context of localization and mapping on metal structures has not been thoroughly investigated. This work aims to answer this need.

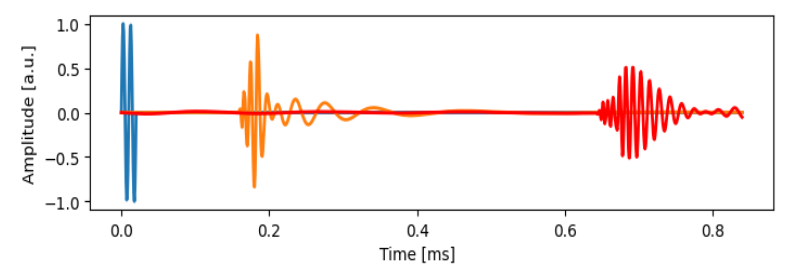

Fig. 2. Illustration of wave dispersion in plates with simulated data. The excitation signal is in blue, the signal propagated after 0.5 meters in orange, and the signal propagated after 2 meters in red.

In typical guided wave data, there are numerous echoes due to the multiple reflections on the plate edges and their number increases exponentially with the observation time. In addition, the wave packets overlap because of the wave dispersion. The consequence is that it is very challenging to recover individual wave-packets from the mixture data [10]. Therefore, most of the recent SHM techniques still rely only on the incident wave packet [3], [4], [11], [12]. For on-plate localization and mapping purposes, however, the retrieval of multiple echoes is essential, as they all provide range-only information to the edges. In the echo detection literature, time-delay estimation techniques have been successfully applied to ultrasound waves in the air [13], [14] but in a non-dispersive context. In [7], we used $\mathcal{L}_{1}$-regularized least squares to retrieve the multiple echoes without taking into account wave dispersion. Here, we rely on a wave propagation model to determine, through correlation with acoustic data, the likelihood of a reflection over a full range of distances to the transducers. In this new setup, the resolution of the difficult echo association problem is no longer required.

Recently, there have been attempts to infer a plate geometry from guided waves data [15]. Yet, non-dispersive propagation models are used, and the sensors are integrated into the structure. In robotics, the most similar problem is room shape reconstruction from acoustic echoes [5], [6]. However, they rely on sound waves propagating in the air without dispersion and do not consider the association problem to determinate the wall from which each echo originates. In [7], we rely on the most likely echo-line association but the overall algorithm is rather complicated due to the map management, and its robustness is limited. Here, from the likelihoods of reflection, we build beamforming maps to estimate the plate shape and limit ourselves to rectangular geometries (which are to be expected in our application). Then, these elements are integrated into a FastSLAM algorithm to achieve localization and mapping simultaneously.

In summary, we present a new method that efficiently integrates wave propagation models from the guided waves theory and beamforming maps in a FastSLAM algorithm to achieve more accurate on-plate localization and mapping results with less algorithmic complexity comparing to our previous method. The results obtained with experimental acoustic data from different metal plates support our claim.

\section{METHOD}

In this work, we are considering a mobile unit equipped with a co-localized emitter/receiver pair of transducers and moving on a metal surface. At the $i^{\text {th }}$ scanning position, the emitter sends a pulse $s(t)$ to excite guided waves in the plate material, and the receiver collects the acoustic response $z_{i}(t)$ which contains the ultrasonic echoes. We intend to use these data and the robot odometry to recover accurately both the plate shape and the robot trajectory.

\section{A. Measurement model}

Acoustic measurements essentially consist in a succession of the reflections of the excitation wave on the plate boundaries. As the small-sized corrosion patches we aim to detect with robotic inspection may not act as reflectors, their potential effect is neglected for the SLAM problem. Under the assumption that the material is isotropic, the propagation linear, and the reflections on the edges are orthogonal, a standard measurement model to reverberation is the image source model [16]. It relies on the fact that each reflection from the plate boundaries can be considered as a signal originating from a fictional source, which is deduced from the real source position and the reverberant media geometry. In metal plates, the image source model can be leveraged to account for first order as well as higher order reflections, resulting in the following measurements:

$$
z_{i}(t)=\sum_{\mathbf{x} \in \mathcal{I}\left(\mathbf{x}_{i}\right)} g\left(\mathbf{x}, \mathbf{x}_{i}, t\right) * s(t)
$$

where $\mathbf{x}_{i}=\left[x_{i}, y_{i}\right]$ is the position of the robot during time step $i, \mathcal{I}\left(\mathbf{x}_{i}\right)$ the set of the image sources positions when the real source is in $\mathbf{x}_{i}, g\left(\mathbf{x}, \mathbf{x}_{i}, t\right)$ the acoustic response of 
the plate to an impulse being generated in $\mathbf{x}$ and received in $\mathbf{x}_{i}$, and $*$ denotes the convolution operation. In a nondispersive media, the impulse response is simply given by $g\left(\mathbf{x}, \mathbf{x}_{i}, t\right)=\delta\left(t-\frac{\left\|\mathbf{x}-\mathbf{x}_{i}\right\|}{c}\right)$, where $\delta$ denotes the Dirac distribution, and $c$ is the constant propagation velocity. It results in waves propagating at a constant speed and without distortion. In a dispersive media like metal plates, a wellsuited model of the propagation is given by the solutions of the Helmholtz equation [17]. For an ideal isotropic media, the impulse response is only a function of the propagation distance $r$ between the (fictional) source and the receiver. Moreover, it is usually reduced, in the Fourier domain, to:

$$
\hat{g}(r, \omega) \approx e^{-j k(\omega) r} / \sqrt{k(\omega) r} .
$$

where $k(\omega)$ is the wavenumber of the major acoustic mode, and its non-linear dependency with respect to the pulsation $\omega$ is the typical characteristic of dispersive propagation in materials. More details on how to determine this relation given prior information on the plate (material, thickness...) can be found in the literature [4].

\section{B. Correlation-based echo detection}

With the aim to retrieve the distances of the robot to the edges from data $z_{i}(t)$, we use the designed propagation model to estimate the likelihood that an orthogonal reflection occurred at a distance $r$. First, we consider the signal that would only contain such a reflection: $\hat{z}(r, t)=\hat{g}(2 r, t) * s(t)$. Next, we build the correlation signal to assess the likelihood that this pattern is present within the measurement:

$$
z_{i}^{\prime}(r)=\frac{\left\langle z_{i}(t), \hat{z}(r, t)\right\rangle}{\sqrt{\left\langle z_{i}(t), z_{i}(t)\right\rangle\langle\hat{z}(r, t), \hat{z}(r, t)\rangle}}
$$

where $\langle.,$.$\rangle denotes the scalar product in the domain of$ continuous signals: $\langle u(t), v(t)\rangle=\int_{-\infty}^{+\infty} u(\tau) v(\tau) d \tau$. As the resulting signal $z_{i}^{\prime}$ presents oscillations consistent with the wave spatial periodicity, it is more convenient to only work with its envelope that we will call $z_{i}(r)$ for simplicity (which shall not be mistaken with the temporal signal $\left.z_{i}(t)\right)$ :

$$
z_{i}(r)=\left|z_{i}^{\prime}(r)+j \mathcal{H}\left(z_{i}^{\prime}\right)(r)\right|
$$

where $\mathcal{H}$ denotes the Hilbert transform operator. Hence, the resulting signal $z_{i}$ takes its values only between 0 and 1 , and a higher value for $r$ translates intofor a high likelihood that a reflection occurred at such a distance. In summary, by looking at the local maxima of $z_{i}(r)$, one can deduce the most likely reflections. Besides, it is noteworthy that a single measurement cannot provide enough information to determine an edge without ambiguity, as all the lines tangent to the circle with radius $r$ and centered to the sensors position may equally account for the correlation measurement.

\section{Map estimation via beamforming}

Similarly to our previous work, the map is represented by a set of lines: $\mathbf{M}=\left\{r_{l}, \theta_{l}\right\}_{l=1 \ldots 4}$ where the parameters $\left(r_{l}, \theta_{l}\right)$ define the line equation in the 2D plane with:

$$
x \cdot \cos \theta_{l}+y \cdot \sin \theta_{l}-r_{l}=0
$$

in a non-mobile frame with respect to the plate. Moreover, as we limit our case-study to rectangular shapes, the possible maps possess only four lines forming a rectangle altogether.

Let's assume a hypothetical robot trajectory $\left\{x_{i}, y_{i}\right\}_{i=1 \ldots T}$. We aim at estimating the map $\mathbf{M}$, which means establishing the probability density function $p\left(\mathbf{M} \mid x_{1 . . T}, y_{1 . . T}, z_{1 \ldots T}\right)$. A first solution would consist in assessing, for each map in the 8-D domain, the correlation between the observations and the predicted data based on the image source model. However, such an approach would be far too cumbersome for a real-time application. Instead, we rely on a beamforming map. Such a map attributes, to every line parameters $(r, \theta)$, the likelihood of the line existence given the observations. It is computed with:

$$
\mathcal{L}_{T}(r, \theta)=\sum_{i=1}^{T} z_{i}\left(\left|x_{i} \cdot \cos \theta+y_{i} \cdot \sin \theta-r\right|\right) .
$$

where $d_{i}(r, \theta)=\left|x_{i} \cdot \cos \theta+y_{i} \cdot \sin \theta-r\right|$ is the distance between the robot during time-step $i$ and the hypothetical line being considered. In the equation, all the correlation values add up constructively along all the observations if an edge is indeed present. Also, it can be noted that only first-order reflections are taken into account, as we reason on individual lines. One may consider that higher order reflections are less likely to account for high correlation amplitudes because of wave scattering after each additional reflection which causes loss of energy to the wave packet. Finally, to retrieve the most plausible map, we solve the following optimization problem:

$$
\hat{\mathbf{M}}=\arg \max _{\mathbf{M}} \mathcal{L}_{T}(\mathbf{M})=\arg \max _{\mathbf{M}} \sum_{l=1}^{4} \mathcal{L}_{T}\left(r_{l}, \theta_{l}\right)
$$

where $\mathbf{M}$ is restricted to be a rectangle. It can be solved efficiently by taking that constraint into account. First, one can determine the most likely line:

$$
\left(r_{1}, \theta_{1}\right)=\arg \max _{r, \theta} \mathcal{L}_{T}(r, \theta) .
$$

Next, it is possible to rely on the assumption that the retrieved line provides the most reliable estimation of the plate orientation w.r.t. the robot. Therefore, the determination of the other lines for $l=2,3,4$ reduces to solving simple and independent one-dimensional optimization problems:

$$
\theta_{l}=\theta_{1}+\frac{\pi(l-1)}{2} ; \quad r_{l}=\arg \max _{r} \mathcal{L}_{T}\left(r, \theta_{l}\right) .
$$

\section{Particle evaluation and FastSLAM algorithm}

FastSLAM is a common approach to approximate Bayesian filters in the context of a SLAM problem. It relies on a particle filter in the localization space, where each particle holds a hypothesis on the map which is inferred from the particle trajectory and the measurements. During time step $T$, a set with $N$ particles has the following form:

$$
\mathcal{P}_{T}=\left\{\mathbf{X}_{T}^{(n)}=\left\{x_{i}^{(n)}, y_{i}^{(n)}, \alpha_{i}^{(n)}\right\}_{i=1 \ldots T}, \mathcal{L}_{T}^{(n)}\right\}_{n=1 \ldots N}
$$

where $\mathbf{X}_{T}^{(n)}$ represents the $n$-th particle belief on the robot trajectory augmented with its heading over time steps $i=$ 
$1 \ldots T$, and $\mathcal{L}_{T}^{(n)}$ its beamforming map which depends on the trajectory. Moreover, each particle is provided with a weight indicating how the particle belief accounts for the measurements. To define it, we rely on the current correlation measurement and assess the likelihoods of the map edges retrieved from $\mathcal{L}_{T}^{(n)}$ and the current robot position belief:

$$
w_{T}^{(n)}=\eta \cdot \exp \left\{\beta \sum_{\left(r_{l}, \theta_{l}\right) \in \mathbf{M}_{T}^{(n)}} z_{T}\left(d_{T}^{(n)}\left(r_{l}, \theta_{l}\right)\right)\right\}
$$

where $\eta$ is the normalization factor and $\beta$ a positive parameter. It enables to fix the confidence in the correlation measurements and shall be tuned so that the resulting weight distribution is consistent with the motion and observation noises. The weights are used to sample, with replacement, the particles after each time step. Besides, one may note that we are not considering, in (4), the uncertainty on the lines retrieval from the beamforming maps for simplicity. Altogether, the implementation of FastSLAM is straightforward and is given in Algorithm 1.

\section{RESULTS}

In this part, we test our FastSLAM approach on experimental data. We detail the experimental setup and show the results in terms of localization and mapping accuracy.

\section{A. Experimental setup}

In order to assess the efficiency of our procedure, we use an emitter-receiver pair of transducers on two different metal plates. The first plate has dimensions $600 \times 450 \times 6 \mathrm{~mm}$, is in aluminium, and has small artificial holes on it. The second plate has dimensions $1700 \times 1000 \times 6 \mathrm{~mm}$ and is in steel. The acoustic data for the plate 1 have been already presented in [7] and will serve as a way to demonstrate the improvement

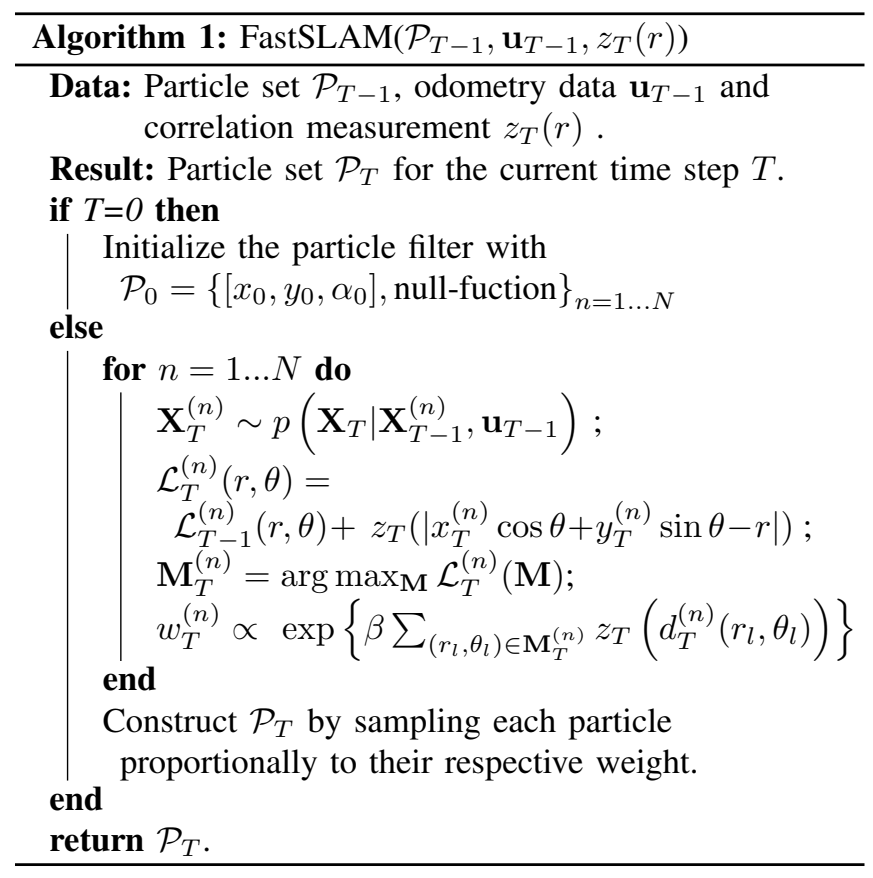

of the procedure. The acquisition process is globally the same to collect the data on the second plate: the transducer pair is moved by hand on the vertices of a regular grid. At every position, 10 measurements of the ultrasonic response are averaged to improve the signal quality. This operation is not critical in a laboratory environment, but it may be necessary in outdoor conditions, where the inspection robot shall operate, to alleviate the effect of external disturbances. The acquisition positions are also carefully recorded. In total, 108 measurements are collected on the plate 1, while this number increases to 117 for plate 2 . We use two tonebursts of a sinusoidal wave at $100 \mathrm{kHz}$ as the excitation. Moreover, the direct incident signal is smoothly removed from the data as it does not correspond to a reflection on an edge.

For each plate, we determine a wave propagation model as in eq. (1) and use $N=20$ particles. To simulate a sweep of a plate by a robotic crawler, a sequence of measurements is selected from the database and is presented to the SLAM framework, with the theoretic displacement between grid cells used as odometry. Also, we add Gaussian noise on the odometry data: $\overline{\Delta r} \sim \mathcal{N}\left(\Delta r,\left(10^{-2} \Delta r+\Delta r_{0}\right)^{2}\right)$ and $\Delta^{-} \theta \sim \mathcal{N}\left(\Delta \theta,\left(10^{-2} \Delta \theta+\Delta \theta_{0}\right)^{2}\right)$ with $\Delta r_{0}=10^{-3} \mathrm{~m}$ and $\Delta \theta_{0}=10^{-2}$ rad to simulate odometry uncertainty which may be limited due to the robot magnetic adherence and embedded accelerometers used to provide precise heading on a nearly-vertical structure, in a realistic scenario [7].

\section{B. Echo detection}

First, we illustrate the echo-detection principle. We show, in Fig.3.a), the measured acoustic signal for a position corresponding to $8 \mathrm{~cm}$ to the edges, in a corner of plate 1 . On b), we show the resulting correlation signal computed using eq. (2) and its envelope calculated with eq. (3), yielding the signal which is fed to the FastSLAM algorithm. It can be seen on $b$ ) that we manage to retrieve, from the local maxima, all the distances where first-order reflections occurred which are 8,37 and $52 \mathrm{~cm}$. The echo detected at nearly $37 \mathrm{~cm}$ corresponds to a second-order reflection, and has a lower amplitude comparing to the first-order wave packets. The existence of such reflections is not assumed by the algorithm. Hence, we will determine a posteriori if their presence has a detrimental effect on the results.

\section{Localization and mapping results}

We run our FastSLAM algorithm using the data of plate 1, and simulate a lawn-mower path. Although the results are generated off-line, our method can run online on a real robotic platform. Indeed, as the beamforming maps of size $Z \times Z$ are updated incrementally, the complexity of one FastSLAM iteration with $N$ particles is $\mathcal{O}\left(N \times Z^{2}\right)$, which leads to a computational time of a few tens of milliseconds per iteration in our setup, with $Z=300$ and $N=20$.

In Fig.4, we show the particles' belief on the sensors trajectory during measurement steps 1, 22, 50 and 108. We also represent the map retrieved by the particle with the highest weight and several dead-reckoning trajectories obtained using noisy odometry input only. During Step 1, the map is not correctly estimated. As only one measurement 


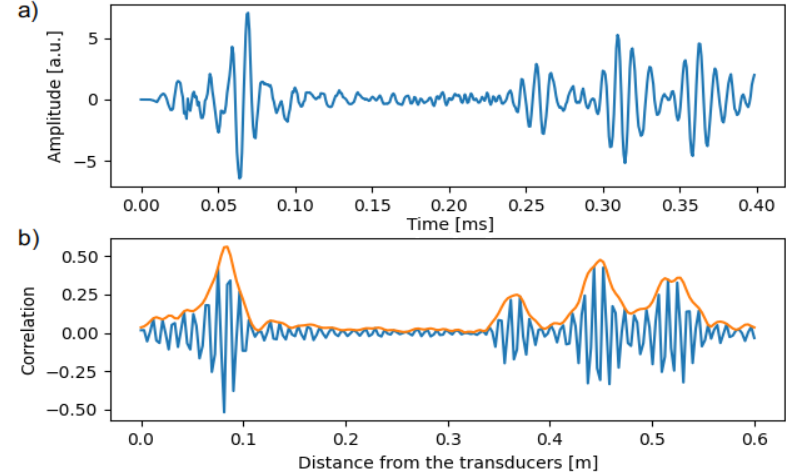

Fig. 3. Illustration of the echo detection principle based on correlation with a propagation model. a) represents the acoustic measurement. b) shows the correlation signal (blue) and its envelope (orange).

has been integrated, the distance to the closest edge can be recovered but, the orientation is essentially random. Rapidly, the three closer edges are recovered as shown during Step 22. However, the right edge is not yet well estimated as it is further away. During Step 50, the plate shape is fully recovered, and during the final step, both the estimation of the plate shape and trajectory are accurate. In contrast, the deadreckoning trajectories present noticeable drift. This illustrates that, by relying on the acoustic data, the proposed approach can appropriately compensate for moderate odometry noise.

Fig.5 depicts the beamforming map for the most likely particle during the final step. We can see that the intensity peaks due to the edges are clearly visible, and our optimization method does not face difficulty to retrieve them.

To compare our new FastSLAM approach with the previous one, we show, in Fig.6, the average localization and line parameters estimation errors calculated over 100 runs of each algorithm, and using the same acoustic data on plate 1 . We simulated 100 repetitions of the lawn-mower path for the sensors trajectory. In the figure, we represent the $10 \%$ and $90 \%$ quantiles with the aim to measure the repeatability of each approach. It can be observed that, with our new

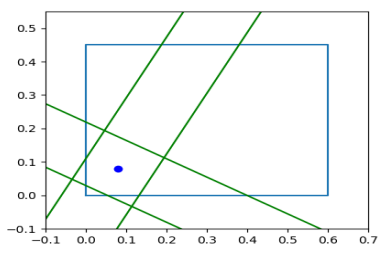

Step 1

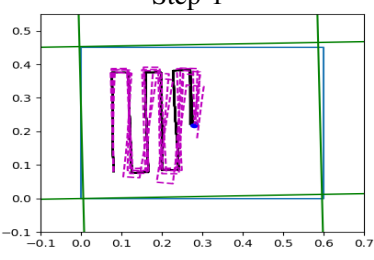

Step 50

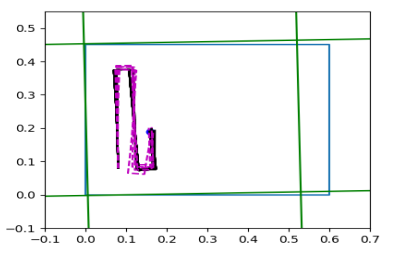

Step 22

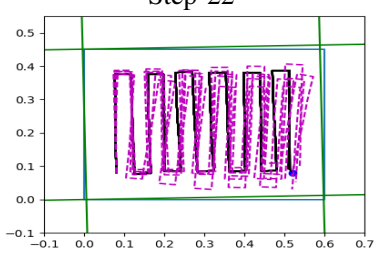

Step 108
Fig. 4. Trajectories estimated by all the particles (black lines), deadreckoning trajectories (dash magenta lines) and map retrieved by the most likely particle (green lines) during Steps 1,22, 50 and 108 for a lawn-mower path on plate 1 (zoom for details). The true outline of the plate and true sensor positions correspond to the blue rectangle and blue dot respectively.

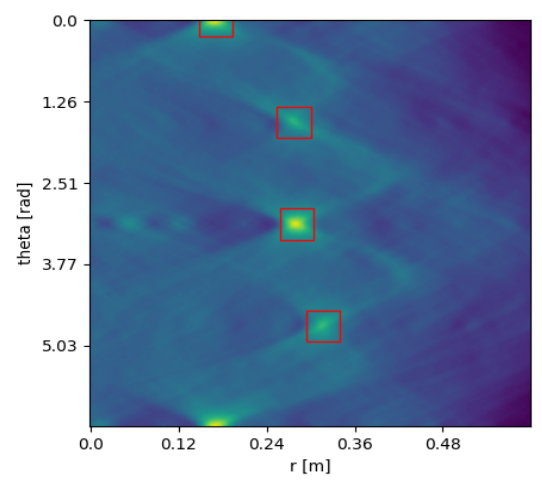

Fig. 5. Beamforming map for the particle with the highest weight during the final step. The red rectangles indicate the edges retrieved with our optimization method.

method, only a few tens of measurement steps are necessary to recover, in average, the range parameters of the lines with a precision of a few millimeters, and the plate orientation with a precision better than one degree. The localization result is also very precise as, after a quick convergence, the position errors remain in the order of a few millimeters despite the defects on the plate. Besides, the estimation is not subject to randomness as the $10 \%$ and $90 \%$ quantiles remain close to the average results. In comparison, our previous method demonstrates poorer results. Indeed, not only are the estimation errors higher, but also the variation of precision can be relatively significant between two runs. Altogether, the results illustrate the improvement of localization and mapping that is achieved by our new method.

With the aim to assess the results for a larger plate, we run our algorithm with the measurements obtained on plate 2 , and simulate again a lawn-mower path. The results obtained over 100 runs are provided in Fig.7. On this plate,
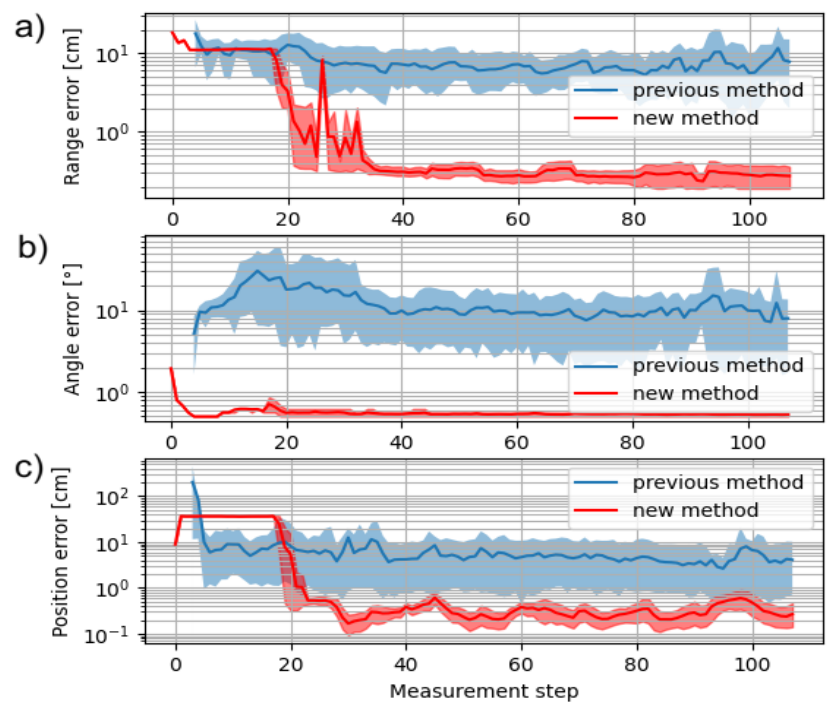

Fig. 6. Localization and mapping results over 100 repetitions of a lawnmower path on plate 1 for the previous and the new method. a) Average estimation errors on the range parameter of the lines. b) Average estimation errors on the angle parameter. c) Average localization errors in the estimated plate frame. The $10 \%$ and $90 \%$ quantiles correspond to the upper and lower bounds of the coloured areas. The scales along the y-axis are logarithmic. 

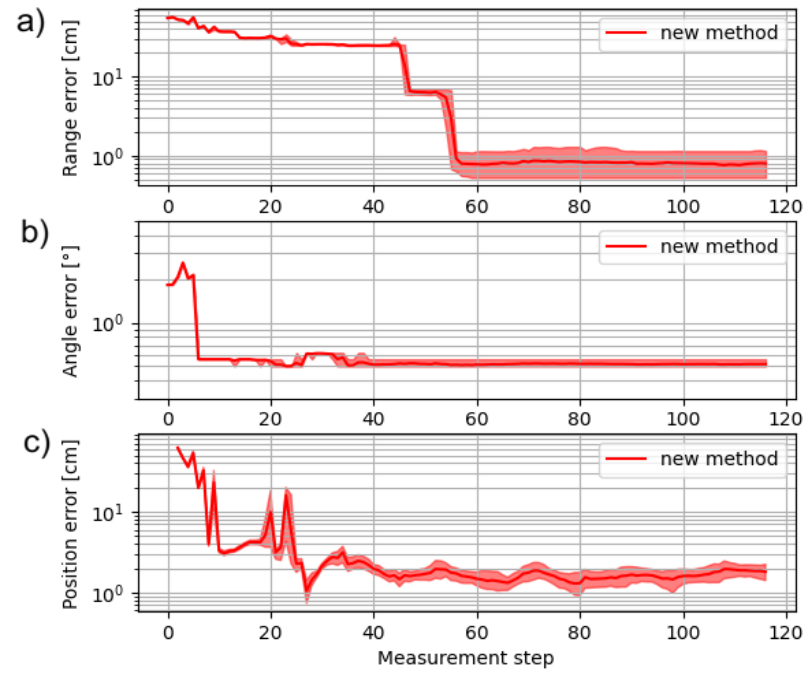

Fig. 7. Localization and mapping results over 100 repetitions of a lawnmower path on plate 2 for the new method. a) Average estimation errors on the range parameter of the lines. b) Average estimation error on the angle parameter. c) Average localization errors in the estimated plate frame. The $10 \%$ and $90 \%$ quantiles correspond to the upper and lower bounds of the coloured areas. The scales along the $y$-axis are logarithmic.

the echo detection employed by our previous method is not efficient, as it does not consider the wave dispersion effect, whereas the propagation distances are larger. This induces large misdetection rates and poor results. Hence, we display only the results of our new approach. Despite the slower convergence caused by the larger surface, and the slightly higher localization error, our method still provides precise estimates of the trajectory and plate geometry. This result indicates that our approach still works on surfaces sufficiently large to be used for realistic applications. The underlying prerequisites are a wave propagation model and filter parameters (number of particles, probabilistic transition model, the parameter $\beta$ ) that conveniently fit the acoustic measurements and on-the-field noisy conditions. Naturally, one may also expect longer convergence times when the plate surface increases, as the echo detection is expected to be efficient mostly for short ranges as shown in Fig.7.a).

As a final evaluation, we determine the average mapping errors and standard deviations over 100 runs obtained during the final step for a lawn-mower path (Scenario 1) and a random walk (Scenario 2) on plate 1. Table 8 presents the results. It can be noticed that the overall results are relatively poorer for the random walk. This illustrates that the estimation accuracy also strongly depends on the robot path which shall be optimized for optimal reconstruction.

\section{CONCLUSIONS}

We have designed a new FastSLAM approach to achieve Simultaneous Localization and Mapping on metal plates by relying on ultrasonic guided waves. Comparing to our previous work, this method relies on wave propagation models and beamforming maps. Experiments carried on an undamaged and a damaged metal plate in a laboratory environment demonstrate that this new approach achieves better results

\begin{tabular}{|l|c|c|}
\hline Scenario & Range error $[\mathrm{mm}]$ & Angle error [degree] \\
\hline Scenario 1 & $3.007 \pm 0.098$ & $0.234 \pm 0.0004$ \\
\hline Scenario 2 & $10.766 \pm 22.921$ & $0.206 \pm 0.134$ \\
\hline
\end{tabular}

Fig. 8. Average estimation errors and standard deviations on the lines parameters obtained during the last measurement step for the two scenarios in consideration. The errors are evaluated using 100 repetitions.

in terms of accuracy and robustness with less algorithmic complexity. In future works, this method shall be adapted and tested in more realistic scenarios. Indeed, on a large metal structure in outdoor environments, more complex and noisy signals are expected due, for example, to inferior surface quality, to the presence of anti-fouling coating on the plates, to more complex plate geometries, or due to wave scattering caused by the welds which fix the different plates altogether. Furthermore, adaptive techniques shall be investigated to adjust the propagation model and filter parameters which may no longer be assumed known a priori. Also, a real robotic platform shall be used, and active-sensing strategies shall be investigated to recover the plate geometry efficiently.

\section{REFERENCES}

[1] M. Montemerlo, S. Thrun, D. Koller, B. Wegbreit et al., "Fastslam: A factored solution to the simultaneous localization and mapping problem," Aaai/iaai, 2002.

[2] W. Cailly and H. Walaszek, "Three dimensional ultrasonic imaging of mechanical components by inversion," in 7th edition of the International Symposium on AirCraft Materials, 2018.

[3] P. Huthwaite and F. Simonetti, "High-resolution guided wave tomography," Wave Motion, 2013.

[4] Z. Su and L. Ye, Identification of Damage Using Lamb Waves: From Fundamentals to Applications, 01 2009, vol. 48.

[5] M. Kreković, I. Dokmanić, and M. Vetterli, "Echoslam: Simultaneous localization and mapping with acoustic echoes," in IEEE International Conference on Acoustics, Speech and Signal Processing, 2016.

[6] F. Peng, T. Wang, and B. Chen, "Room shape reconstruction with a single mobile acoustic sensor," in 2015 IEEE Global Conference on Signal and Information Processing (GlobalSIP). IEEE, 2015.

[7] C. Pradalier, O.-L. Ouabi, P. Pomarede, and J. Steckel, "On-plate localization and mapping for an inspection robot using ultrasonic guided waves: a proof of concept," in IROS. IEEE, 2020.

[8] B. D. Van Veen and K. M. Buckley, "Beamforming: a versatile approach to spatial filtering," IEEE ASSP Magazine, 1988.

[9] P. Cawley and D. Alleyne, "The use of lamb waves for the long range inspection of large structures," Ultrasonics, 1996.

[10] Z. Su, L. Ye, and Y. Lu, "Guided lamb waves for identification of damage in composite structures: A review," Journal of Sound and Vibration, vol. 295, no. 3, pp. 753 - 780, 2006.

[11] M. Zhao, W. Zhou, Y. Huang, and H. Li, "Sparse bayesian learning approach for propagation distance recognition and damage localization in plate-like structures using guided waves," Structural Health Monitoring, vol. 0, no. 0, p. 1475921720902277, 0. [Online]. Available: https://doi.org/10.1177/1475921720902277

[12] Q. Jianxi, F. Li, S. Abbas, and Y. Zhu, "A baseline-free damage detection approach based on distance compensation of guided waves," Journal of Low Frequency Noise, Vibration and Active Control, 2018.

[13] J. Steckel and H. Peremans, "Sparse decomposition of in-air sonar images for object localization," in Proceedings of IEEE Sensors, vol. 2014-Decem, no. December, 2014.

[14] B. Fontaine and H. Peremans, "Determining biosonar images using sparse representations." The Journal of the Acoustical Society of America, vol. 125, no. 5, pp. 3052-9, may 2009.

[15] E. Hong and C. Schaal, "Reverse engineering stiffened plates using guided wave-based nondestructive testing methods ," in Health Monitoring of Structural and Biological Systems XII, T. Kundu, Ed., International Society for Optics and Photonics. SPIE, 2018.

[16] H. Kuttruff, Room Acoustics, fourth edition, 2000.

[17] N. Quaegebeur, P. Masson, D. Langlois Demers, and P. Micheau, "Dispersion-based imaging for structural health monitoring using sparse and compact arrays," Smart Materials and Structures, 2011. 